\title{
$X(3872)$ in an unquenched quark model
}

\author{
Yue Tan ${ }^{*}$ and Jialun Ping ${ }^{\dagger}$ \\ Department of Physics and Jiangsu Key Laboratory for Numerical Simulation of Large Scale \\ Complex Systems, Nanjing Normal University, Nanjing 210023, People's Republic of China
}

(Received 4 July 2019; published 23 August 2019)

\begin{abstract}
In this paper we calculate the mass and probability fractions of the meson-meson components of $X(3872)$ in an unquenched quark model. Different from most other unquenched quark models, the quarkpair creation operator from ${ }^{3} P_{0}$ is modified by considering the effects of the created quark pair's energy and the separation between the created quark pair and the valence quark pair. In the calculation all of the wave functions of the mesons and the relative motion between two mesons are obtained by solving the corresponding Schrödinger equation with the help of the Gaussian expansion method. The multichannel couplings of the quark-antiquark state with possible meson-meson states are calculated. The results show that $X(3872)$ can be described as a mixing state of the dominant charmonium state $(70 \%)$ and mesonmeson components $(30 \%)$.
\end{abstract}

DOI: $10.1103 /$ PhysRevD.100.034022

\section{INTRODUCTION}

The nonrelativistic quark model successfully describes the properties of heavy mesons. However, since the wellknown exotic state $X(3872)$ was discovered more and more exotic particles that cannot fit well in the meson spectrum have been reported by experimental collaborations. These exotic states represent a great challenge for the quark model.

In 2003, the Belle Collaboration first found $X(3872)$ in $B$-meson decays [1]. Subsequently, the CDF [2] and D0 [3] collaborations confirmed this state in $p \bar{p}$ collisions, and $B A B A R$ also found $X(3872)$ in $B$-meson decays [4]. Different from the ordinary hadrons, the state $X(3872)$ has some strange properties: its mass is very close to the threshold of $D \bar{D}^{*}$, and its decay width is very narrow (less than $1.2 \mathrm{MeV})$. So the nature of $X(3872)$ is still an open question. Due to the ambiguous information about the quantum numbers of $X(3872)$ at the time, some held the view that the traditional quark model can still describe its properties [5-8]. Barnes et al. analyzed some states by calculating their radiative transitions, and it turned out that five states $\left(1^{3} D_{3}, 1^{3} D_{2}, 1^{1} D_{2}, 2^{3} P_{1}\right.$, and $\left.2^{1} P_{1}\right)$ could be possible candidates for the new exotic state [5].

\footnotetext{
*161002010@stu.njnu.edu.cn

Corresponding author. jlping@njnu.edu.cn

Published by the American Physical Society under the terms of the Creative Commons Attribution 4.0 International license. Further distribution of this work must maintain attribution to the author(s) and the published article's title, journal citation, and DOI. Funded by SCOAP ${ }^{3}$.
}

After examining the models of mesons and meson-meson molecules, Swanson et al. and Pakasa and Suzuki concluded that the meson picture may be more suitable for $X(3872)$ [6,9]. Indeed, the ratio $\frac{B(X(3872) \rightarrow \psi(2 s) \gamma)}{B(X(3872) \rightarrow J / \psi \gamma)}=3.4 \pm$ 1.4 announced by the BABAR Collaboration appears to support the claim that $X(3872)$ is a traditional meson [10]. Achasov argued that the $X(3872)$ resonance was the $c \bar{c}=$ $\chi_{c 1}(2 P)$ charmonium which "sits on" the $D^{* 0} \bar{D}^{0}$ threshold, and its mass was shifted from the prediction of the potential model to the threshold of $D^{*} \bar{D}$ by the contribution of the virtual $D^{*} \bar{D}+$ c.c. intermediate states [11].

Due to the fact that the mass of $X(3872)$ is close to the threshold of $D \bar{D}^{*}$, it is natural to interpret the state $X(3872)$ in the molecular picture [7]. Törnqvist proposed that, analogously to the deuteron, the one-pion-exchange potential could make a contribution to the bound state of $X(3872)$ [7], and that $X(3872)$ has the structure $\frac{1}{\sqrt{2}}\left[D \bar{D}^{*}+\bar{D} D^{*}\right]$. This picture could easily explain the isospin-breaking branching ratio $\frac{\operatorname{Br}\left(J / \psi \pi^{-} \pi^{+} \pi^{0}\right)}{\operatorname{Br}\left(J / \psi \pi^{+} \pi^{-}\right)}=1.0 \pm$ $0.4 \pm 0.3$ [12] due to the mass difference between the neutral $D$ meson and charged $D$ meson. Based on the pion exchange potential proposed by Törnqvist, Swanson et al. added an additional mixture of $J / \psi \rho$ and $J / \psi \omega$ to the state, and concluded that although the effect of pion exchange might be responsible for the bound state, the short-range quark dynamics were present and assisted in binding the $X(3872)$ via mixing to a hidden charm vector $(J / \psi$ states $)$ [13]. However, some believed that pion exchange was too weak to bind $X(3872)[8,14,15]$. Because $D^{*}-D-\pi=0$, Suzuki thought that there was no long-range attraction between $D$ and $D^{*}$, which means that $X(3872)$ is not a 
bound state [8]. In addition, unless the coupling constants and cutoffs were large, $X(3872)$ could not be a real molecule that only depends on the pion exchange potential [14]. Thomas et al. added a tensor tern and a flavor factor to the one pion exchange model, and they finally got a bound state [15].

Actually, since the $B A B A R$ Collaboration reported the branching ratio of $X \rightarrow \gamma J / \psi$, people have started to accept the concept that $X(3872)$ might be an unquenched hadron state-a mixture of $c \bar{c}$ and $D \bar{D}^{*}$ [16-19]. Kang and Oller analyzed the experimental data using a near-threshold parametrization method and found that the $X(3872)$ compositeness coefficients in $D^{0} \bar{D}^{* 0}$ range from nearly 0 up to 1 in the different scenarios [20]. In 2005, Kalashnikova first used the ${ }^{3} P_{0}$ model to study the mass spectrum of $c \bar{c}$ based on the nonrelativistic quark model, in which the simple harmonic oscillator (SHO) functions are used to describe the wave functions of mesons, and obtained an $X(3872)$ mass of $3990 \mathrm{MeV}$ [16], only $0.3 \mathrm{MeV}$ over threshold. In the following years, the $q \bar{q} \rightarrow q q \bar{q} \bar{q}$ transition operator in the unquenched quark model (UQM) was often taken as the ${ }^{3} P_{0}$ operator, which was first proposed by Micu [21]. Then, Yaouanc et al. used the operator to calculate the strong decay widths of baryons and mesons [22,23]. Santopinto et al. calculated the decay width, components, and mass of $X(3872)$ using the relativistic quark model and ${ }^{3} P_{0}$ model, where SHO functions were also used [17,24-28]. In addition, Ortega et al. utilized the unquenched quark model to analyze the decay width and components of $X(3872)$, and their wave functions for the relative motion between two mesons were obtained by solving the resonating group method equation [18,29-31]. In many previous works the SHO functions are used to describe the meson dynamics and the relative motion between two mesons is described by plane-wave functions. The systematic errors due to the approximations are unpredictable for the bound-state calculation, although they are not a bad approximation for the decay width calculation [32]. In this work we apply the Gaussian expansion method (GEM) - which is a powerful method for few-body systems- to analyze $X(3872)$ in an unquenched quark model [32] with a modified ${ }^{3} P_{0}$ operator.

The paper is organized as follows. In Sec. II the chiral quark model and GEM for solving the $q \bar{q}$ and $q \bar{q}-q \bar{q}$ systems are presented. In Sec. III we briefly introduce the modified ${ }^{3} P_{0}$ model. The numerical results are given in Sec. IV. The last section is devoted to the summary of the present work.

\section{CHIRAL QUARK MODEL AND GEM}

In the chiral quark model [33], the meson spectrum is obtained by solving the Schrödinger equation

$$
H \Psi_{I M_{I}}^{J M_{J}}(1,2)=E^{I J} \Psi_{I M_{I}}^{J M_{J}}(1,2) .
$$

The wave function $\Psi_{I M_{I}}^{J M_{J}}$ of a meson with quantum numbers $I^{G} J^{P C}$ can be written as

$\Psi_{I M_{I}}^{J M_{J}}(1,2)=\sum_{\alpha}\left[\psi_{l}(\mathbf{r}) \chi_{s}(1,2)\right]^{J M_{J}} \omega^{c}(1,2) \phi_{I M_{I}}(1,2)$,

where $\alpha$ denotes the intermediate quantum numbers $l, s$ and possible flavor indices (for isospin $I=0$ states, the flavor indices are $u \bar{u}, d \bar{d}$, and $s \bar{s})$. "[ ]" denotes the angular momentum coupling, $\chi_{s m_{s}}(1,2), \omega^{c}(1,2)$, and $\phi_{I M_{I}}(1,2)$ are the spin, color, and flavor wave functions (with specific isospin $I$ ) of the meson, respectively, and $\psi_{l m}(\mathbf{r})$ (where $\mathbf{r}=\mathbf{r}_{1}-\mathbf{r}_{2}$ ) is the orbital wave function. In GEM, the orbital wave function is written as the product of a radial function and spherical harmonics, and the radial part of the wave function is expanded by a set of Gaussians,

$$
\begin{aligned}
\psi_{l m}(\mathbf{r}) & =\sum_{n=1}^{n_{\max }} c_{n} \psi_{n l m}^{G}(\mathbf{r}), \\
\psi_{n l m}^{G}(\mathbf{r}) & =N_{n l} r^{l} e^{-\nu_{n} r^{2}} Y_{l}^{m}(\hat{\mathbf{r}}) .
\end{aligned}
$$

The Gaussian size parameters are taken as the following geometric progression numbers:

$\nu_{n}=\frac{1}{r_{n}^{2}}, \quad r_{n}=r_{1} a^{n-1}, \quad a=\left(\frac{r_{n_{\max }}}{r_{1}}\right)^{\frac{1}{n_{\max }-1}}$.

This enables the optimization of the expansion employing a small number of Gaussians. So, the wave function takes the form

$$
\begin{aligned}
\Psi_{I M_{I}}^{J M}(1,2) & =\sum_{n \alpha} C_{n \alpha}^{I J} \Phi_{I M_{I}, n \alpha}^{J M} \\
& =\sum_{n \alpha} C_{n \alpha}^{I J}\left[\Psi_{l}(\mathbf{r}) \chi_{s}\right]^{J M} \omega^{c} \phi_{I M_{I}} .
\end{aligned}
$$

Noting that the Gaussians are not orthogonal, the RayleighRitz variational principle for solving the Schrödinger equation leads to a generalized eigenvalue problem,

$$
\begin{gathered}
\sum_{n^{\prime}, \alpha^{\prime}}\left(H_{n \alpha, n^{\prime} \alpha^{\prime}}^{I J}-E^{I J} N_{n \alpha, n^{\prime} \alpha^{\prime}}^{I J}\right) C_{n^{\prime} \alpha^{\prime}}^{I J}=0, \\
H_{n \alpha, n^{\prime} \alpha^{\prime}}^{I J}=\left\langle\Phi_{I M_{I}, n \alpha}^{J M}|H| \Phi_{I M_{I}, n^{\prime} \alpha^{\prime}}^{J M}\right\rangle, \\
N_{n \alpha, n^{\prime} \alpha^{\prime}}^{I J}=\left\langle\Phi_{I M_{I}, n \alpha}^{J M} \mid \Phi_{I M_{I}, n^{\prime} \alpha^{\prime}}^{J M}\right\rangle .
\end{gathered}
$$

Extended to the $q \bar{q}-q \bar{q}$ system, the same Schrödinger equation is employed to obtain the energy of the system,

$$
H \Psi_{I M_{I}}^{J M_{J}}(1234)=E^{I J} \Psi_{I M_{I}}^{J M_{J}}(1234),
$$

where $\Psi_{I M_{I}}^{J M_{J}}$ is the wave function of the four-quark state, which can be constructed as follows. First, we write down the wave functions of two subclusters, 


$$
\begin{aligned}
& \Psi_{I_{1} M_{I_{1}}}^{J_{1} M_{J_{1}}}(12)=\left[\psi_{l_{1}}\left(\mathbf{r}_{12}\right) \chi_{s_{1}}(12)\right]^{J_{1} M_{J_{1}}} \omega^{c_{1}}(12) \phi_{I_{1} M_{I_{1}}}(12), \\
& \Psi_{I_{2} M_{I_{2}}}^{J_{2} M_{J_{2}}}(34)=\left[\psi_{l_{2}}\left(\mathbf{r}_{34}\right) \chi_{s_{2}}(34)\right]^{J_{2} M_{J_{2}}} \omega^{c_{2}}(34) \phi_{I_{2} M_{I_{2}}}(34),
\end{aligned}
$$

where $\chi_{s}, \omega^{c}$, and $\phi^{I}$ are the spin, color, and flavor wave functions of the quark-antiquark subcluster, respectively (the quarks and antiquarks are numbered as 1, 3 and 2, 4, respectively). The bracket [ ] denotes the angular momentum coupling. Then, the total wave function of the fourquark state is obtained as

$$
\begin{gathered}
\Psi_{I M_{I}}^{J M_{J}}(1234)=\mathcal{A}\left[\left[\psi_{l_{1}}\left(\mathbf{r}_{12}\right) \chi_{s_{1}}(12)\right]^{J_{1}}\right. \\
\left.\left[\psi_{l_{2}}\left(\mathbf{r}_{34}\right) \chi_{s_{2}}(34)\right]^{J_{2}} \psi_{L_{r}}\left(\mathbf{r}_{1234}\right)\right]^{J M_{J}} \\
{\left[\omega^{c_{1}}(12) \omega^{c_{2}}(34)\right]^{[222]}\left[\phi_{I_{1}}(12) \phi_{I_{2}}(34)\right]_{I M_{I}},}
\end{gathered}
$$

where $\psi_{L_{r}}\left(\mathbf{r}_{1234}\right)$ is the wave function for the relative motion between two clusters with orbital angular momentum $L_{r} . \mathcal{A}$ is the antisymmetrization operator. If all quarks (antiquarks) are taken as identical particles, we have

$$
\mathcal{A}=\frac{1}{2}\left(1-P_{13}-P_{24}+P_{13} P_{24}\right) .
$$

The radial part of the wave function is also expanded by Gaussians as in Eq. (3). Finally, the infinitesimally shifted Gaussian basis function is employed for the orbital wave functions with nonzero orbital angular momentum to simplify the calculation of the matrix elements [34]:

$$
\begin{aligned}
\psi_{n l m}^{G}(\mathbf{r}) & =N_{n l} r^{l} e^{-\nu_{n} r^{2}} Y_{l}^{m}(\hat{\mathbf{r}}) \\
& =N_{n l} \lim _{\epsilon \rightarrow 0} \frac{1}{\epsilon^{l}} \sum_{k}^{k_{\max }} C_{l m, k} e^{-\nu_{n}\left(\mathbf{r}-\epsilon \mathbf{D}_{l m, k}\right)^{2}} .
\end{aligned}
$$

The Hamiltonian of the chiral quark model includes three parts: the rest masses of the quarks, the nonrelativistic kinetic energy, and the potential energy. The potential energy is composed of color confinement, one-gluonexchange (OGE), and one-Goldstone-boson-exchange terms. The detailed form of the four-quark states is shown below [33]:

$$
\begin{aligned}
H= & \sum_{i=1}^{4} m_{i}+\frac{p_{12}^{2}}{2 \mu_{12}}+\frac{p_{34}^{2}}{2 \mu_{34}}+\frac{p_{1234}^{2}}{2 \mu_{1234}} \\
& +\sum_{i<j=1}^{4}\left(V_{i j}^{G}+V_{i j}^{C}+\sum_{\chi=\pi, K, \eta} V_{i j}^{\chi}+V_{i j}^{\sigma}\right), \\
V_{i j}^{G}= & \frac{\alpha_{s}}{4} \lambda_{i}^{c} \cdot \lambda_{j}^{c}\left[\frac{1}{r_{i j}}-\frac{2 \pi}{3 m_{i} m_{j}} \boldsymbol{\sigma}_{i} \cdot \boldsymbol{\sigma}_{j} \delta\left(\mathbf{r}_{i j}\right)\right], \\
\delta\left(\mathbf{r}_{i j}\right)= & \frac{e^{-r_{i j} / r_{0}\left(\mu_{i j}\right)}}{4 \pi r_{i j} r_{0}^{2}\left(\mu_{i j}\right)}, \\
V_{i j}^{C}= & \left(-a_{c} r_{i j}^{2}-\Delta\right) \lambda_{i}^{c} \cdot \lambda_{j}^{c},
\end{aligned}
$$

$$
\begin{aligned}
V_{i j}^{\pi}= & \frac{g_{\mathrm{ch}}^{2}}{4 \pi} \frac{m_{\pi}^{2}}{12 m_{i} m_{j}} \frac{\Lambda_{\pi}^{2}}{\Lambda_{\pi}^{2}-m_{\pi}^{2}} m_{\pi} v_{i j}^{\pi} \sum_{a=1}^{3} \lambda_{i}^{a} \lambda_{j}^{a}, \\
V_{i j}^{K}= & \frac{g_{\mathrm{ch}}^{2}}{4 \pi} \frac{m_{K}^{2}}{12 m_{i} m_{j}} \frac{\Lambda_{K}^{2}}{\Lambda_{K}^{2}-m_{K}^{2}} m_{K} v_{i j}^{K} \sum_{a=4}^{7} \lambda_{i}^{a} \lambda_{j}^{a}, \\
V_{i j}^{\eta}= & \frac{g_{\mathrm{ch}}^{2}}{4 \pi} \frac{m_{\eta}^{2}}{12 m_{i} m_{j}} \frac{\Lambda_{\eta}^{2}}{\Lambda_{\eta}^{2}-m_{\eta}^{2}} m_{\eta} v_{i j}^{\eta} \\
& {\left[\lambda_{i}^{8} \lambda_{j}^{8} \cos \theta_{P}-\lambda_{i}^{0} \lambda_{j}^{0} \sin \theta_{P}\right], } \\
V_{i j}^{\sigma}= & -\frac{g_{\mathrm{ch}}^{2}}{4 \pi} \frac{\Lambda_{\sigma}^{2}}{\Lambda_{\sigma}^{2}-m_{\sigma}^{2}} m_{\sigma}\left[Y\left(m_{\sigma} r_{i j}\right)-\frac{\Lambda_{\sigma}}{m_{\sigma}} Y\left(\Lambda_{\sigma} r_{i j}\right)\right], \\
v_{i j}^{\chi}= & {\left[Y\left(m_{\chi} r_{i j}\right)-\frac{\Lambda_{\chi}^{3}}{m_{\chi}^{3}} Y\left(\Lambda_{\chi} r_{i j}\right)\right] \sigma_{i} \cdot \sigma_{j}, } \\
Y(x)= & e^{-x} / x,
\end{aligned}
$$

where $m_{i}$ is the mass of quarks and antiquarks, $\mu_{i j}$ is their reduced mass, $r_{0}\left(\mu_{i j}\right)=\hat{r}_{0} / \mu_{i j}, \sigma$ are the $S U(2)$ Pauli matrices, $\lambda$ and $\lambda^{c}$ are the $S U(3)$ flavor and color GellMann matrices, and $g_{\mathrm{ch}}^{2} / 4 \pi$ is the chiral coupling constant, determined from the $\pi$-nucleon coupling constant. $\alpha_{s}$ is the effective scale-dependent running quark-gluon coupling constant [33],

$$
\alpha_{s}\left(\mu_{i j}\right)=\frac{\alpha_{0}}{\ln \left[\left(\mu_{i j}^{2}+\mu_{0}^{2}\right) / \Lambda_{0}^{2}\right]} .
$$

The quadratic confinement without color screening is employed to avoid the double counting of the contributions from the open four-quark channels [35,36]. All of the parameters are determined by fitting the meson spectrumfrom light to heavy - taking into account only the quarkantiquark component. They are shown in Table I.

TABLE I. Quark model parameters.

\begin{tabular}{lcc}
\hline \hline Quark masses & $m_{u}=m_{d}(\mathrm{MeV})$ & 313 \\
& $m_{s}(\mathrm{MeV})$ & 536 \\
& $m_{c}(\mathrm{MeV})$ & 1728 \\
Goldstone bosons & $m_{b}(\mathrm{MeV})$ & 5112 \\
& $m_{\pi}\left(\mathrm{fm}^{-1}\right)$ & 0.70 \\
& $m_{\sigma}\left(\mathrm{fm}^{-1}\right)$ & 3.42 \\
& $m_{\eta}\left(\mathrm{fm}^{-1}\right)$ & 2.77 \\
& $m_{K}\left(\mathrm{fm}^{-1}\right)$ & 2.51 \\
& $\Lambda_{\pi}=\Lambda_{\sigma}\left(\mathrm{fm}^{-1}\right)$ & 4.2 \\
& $\Lambda_{\eta}=\Lambda_{K}\left(\mathrm{fm}^{-1}\right)$ & 5.2 \\
& $g_{\mathrm{ch}}^{2} /(4 \pi)$ & 0.54 \\
Confinement & $\theta_{p}\left({ }^{\circ}\right)$ & -15 \\
& $a_{c}(\mathrm{MeV})$ & 101 \\
OGE & $\Delta(\mathrm{MeV})$ & -78.3 \\
& $\alpha_{0}$ & 3.67 \\
& $\left.\Lambda_{0}(\mathrm{fm})^{-1}\right)$ & 0.033 \\
& $\mu_{0}(\mathrm{MeV})$ & 36.976 \\
\hline \hline & $\hat{r}_{0}(\mathrm{MeV})$ & 28.17 \\
\hline
\end{tabular}




\section{III. ${ }^{3} P_{0}$ MODEL}

\section{A. Traditional ${ }^{3} \boldsymbol{P}_{\mathbf{0}}$ operator}

The ${ }^{3} P_{0}$ model (quark-pair creation model) was originally introduced by Micu [21] and further developed in Refs. $[22,23,37,38]$. It can be applied to the OZI-ruleallowed two-body strong decays of a hadron [21,37,39-41]. The transition operator in the ${ }^{3} P_{0}$ model is

$$
\begin{aligned}
T_{1}= & -3 \gamma \sum_{m}\langle 1 m 1-m \mid 00\rangle \int d \mathbf{p}_{3} d \mathbf{p}_{4} \delta^{3}\left(\mathbf{p}_{3}+\mathbf{p}_{4}\right) \\
& \times \mathcal{Y}_{1}^{m}\left(\frac{\mathbf{p}_{3}-\mathbf{p}_{4}}{2}\right) \chi_{1-m}^{34} \phi_{0}^{34} \omega_{0}^{34} b_{3}^{\dagger}\left(\mathbf{p}_{3}\right) d_{4}^{\dagger}\left(\mathbf{p}_{4}\right),
\end{aligned}
$$

where $\gamma$ represents the probability of the quark-antiquark pair with momentum $\mathbf{p}_{3}$ and $\mathbf{p}_{4}$ created from the vacuum.
Because the intrinsic parity of the antiquark is negative, the created quark-antiquark pair must be in the state ${ }^{2 S+1} L_{J}={ }^{3} P_{0} . \phi_{0}^{34}$ and $\omega_{0}^{34}$ are flavor- and color-singlet states, respectively (the indices of the quark and antiquark in the original meson are 1 and 2). The $S$-matrix element for the process $A \rightarrow B+C$ is written as

$$
\langle B C|T| A\rangle=\delta^{3}\left(\mathbf{P}_{A}-\mathbf{P}_{B}-\mathbf{P}_{C}\right) \mathcal{M}^{M_{J_{A}} M_{J_{B}} M_{J_{C}}},
$$

where $\mathbf{P}_{B}$ and $\mathbf{P}_{C}$ are the momenta of mesons $B$ and $C$ in the final state, which satisfy $\mathbf{P}_{A}=\mathbf{P}_{B}+\mathbf{P}_{C}=0$ in the center-of-mass frame of meson $A . \mathcal{M}^{M_{J_{A}} M_{J_{B}} M_{J_{C}}}$ is the helicity amplitude of the process $A \rightarrow B+C$, which can be obtained as

$$
\begin{aligned}
\mathcal{M}^{M_{J_{A}} M_{J_{B}} M_{J_{C}}(\mathbf{P})=} & \gamma \sqrt{8 E_{A} E_{B} E_{C}} \sum_{\begin{array}{c}
M_{L_{A}}, M_{S_{A}}, \\
M_{L_{B}}, M_{S_{B}}, \\
M_{L_{C}}, M_{S_{C}}, m
\end{array}}\left\langle L_{A} M_{L_{A}} S_{A} M_{S_{A}} \mid J_{A} M_{J_{A}}\right\rangle\left\langle L_{B} M_{L_{B}} S_{B} M_{S_{B}} \mid J_{B} M_{J_{B}}\right\rangle \\
& \times\left\langle L_{C} M_{L_{C}} S_{C} M_{S_{C}} \mid J_{C} M_{J_{C}}\right\rangle\langle 1 m 1-m \mid 00\rangle\left\langle\chi_{S_{B} M_{S_{B}}}^{14} \chi_{S_{C} M_{S_{C}}}^{32} \mid \chi_{S_{A} M_{S_{A}}}^{12} \chi_{1-m}^{34}\right\rangle \\
& \times\left[\left\langle\phi_{B}^{14} \phi_{C}^{32} \mid \phi_{A}^{12} \phi_{0}^{34}\right\rangle \mathcal{I}_{M_{L_{B}}, M_{L_{C}}}^{M_{L_{C}}, m}\left(\mathbf{P}, m_{1}, m_{2}, m_{3}\right)\right. \\
& \left.+(-1)^{1+S_{A}+S_{B}+S_{C}}\left\langle\phi_{B}^{32} \phi_{C}^{14} \mid \phi_{A}^{12} \phi_{0}^{34}\right\rangle \mathcal{I}_{M_{L_{B}}, M_{L_{C}}}^{M_{L_{A}}, m}\left(-\mathbf{P}, m_{2}, m_{1}, m_{3}\right)\right],
\end{aligned}
$$

with the momentum-space integral

$$
\mathcal{I}_{M_{L_{B}}, M_{L_{C}}}^{M_{L_{1}}, m}\left(\mathbf{P}, m_{1}, m_{2}, m_{3}\right)=\int d \mathbf{p} \psi_{n_{B} L_{B} M_{L_{B}}}^{*}\left(\frac{m_{3}}{m_{1}+m_{3}} \mathbf{P}+\mathbf{p}\right) \psi_{n_{C} L_{C} M_{L_{C}}}^{*}\left(\frac{m_{3}}{m_{2}+m_{3}} \mathbf{P}+\mathbf{p}\right) \psi_{n_{A} L_{A} M_{L_{A}}}(\mathbf{P}+\mathbf{p}) \mathcal{Y}_{1}^{m}(\mathbf{p}),
$$

where $\mathbf{P}=\mathbf{P}_{B}=-\mathbf{P}_{C}, \mathbf{p}=\mathbf{p}_{3}$, and $m_{3}$ is the mass of the created quark $q_{3}$. To analyze the results and compare the theoretical results with experimental data, the partial-wave amplitude $\mathcal{M}^{J L}(A \rightarrow B C)$ is often employed. It is related to the helicity amplitude by the Jacob-Wick formula [42],

$$
\begin{aligned}
\mathcal{M}^{J L}(A \rightarrow B C)= & \frac{\sqrt{2 L+1}}{2 J_{A}+1} \sum_{M_{J_{B}}, M_{J_{C}}}\left\langle L 0 J M_{J_{A}} \mid J_{A} M_{J_{A}}\right\rangle \\
& \times\left\langle J_{B} M_{J_{B}} J_{C} M_{J_{C}} \mid J M_{J_{A}}\right\rangle \mathcal{M}^{M_{J_{A}} M_{J_{B}} M_{J_{C}}(\mathbf{P}) .}
\end{aligned}
$$

In evaluating the momentum space integral (18), we use the wave functions of mesons $A, B, C$ obtained in the mass spectrum calculation. Because the wave functions are expanded by a series of Gaussians, the integral can be evaluated analytically.

The parameter $\gamma$ is generally determined by an overall fitting of the strong decay width of hadrons. In this way, one obtains $\gamma=6.95$ for $u \bar{u}$ and $d \bar{d}$ pair creation, and $\gamma=$ $6.95 / \sqrt{3}$ for $s \bar{s}$ pair creation [43].

\section{B. Modified ${ }^{3} \boldsymbol{P}_{0}$ operator}

The modified transition operator $T_{2}$ (in position space) was first proposed by Chen et al. to deal with the fact that the mass shift of a light meson is too large if the traditional transition operator $T_{1}$ is used [32],

$$
\begin{aligned}
T_{2}= & -3 \gamma \sum_{m}\langle 1 m 1-m \mid 00\rangle \int d \mathbf{r}_{3} d \mathbf{r}_{4}\left(\frac{1}{2 \pi}\right)^{\frac{3}{2}} i r 2^{-\frac{5}{2}} f^{-5} \\
& \times Y_{1 m}(\hat{\mathbf{r}}) e^{-\frac{r^{2}}{4 f^{2}}} e^{-\frac{R_{A V}^{2}}{f_{0}^{2}}} \chi_{1-m}^{34} \phi_{0}^{34} \omega_{0}^{34} b_{3}^{\dagger}\left(\mathbf{r}_{3}\right) d_{4}^{\dagger}\left(\mathbf{r}_{4}\right) .
\end{aligned}
$$

Here, $\mathbf{R}_{A V}=\mathbf{R}_{A}-\mathbf{R}_{V}$ is the relative coordinate between the source particle " $A$ " and the created quark-antiquark pair in the vacuum, with

$$
\begin{aligned}
& \mathbf{R}_{A}=\frac{m_{1} \mathbf{r}_{\mathbf{1}}+m_{2} \mathbf{r}_{\mathbf{2}}}{m_{1}+m_{2}}, \\
& \mathbf{R}_{V}=\frac{m_{3} \mathbf{r}_{\mathbf{3}}+m_{4} \mathbf{r}_{\mathbf{4}}}{m_{3}+m_{4}}=\frac{\mathbf{r}_{\mathbf{3}}+\mathbf{r}_{\mathbf{4}}}{2} .
\end{aligned}
$$


The convergence factor $\exp \left(-r^{2} /\left(4 f^{2}\right)\right)\left[\exp \left(-f^{2} p^{2}\right)\right.$ in momentum space, where $p$ is the relative momentum between the created quark and antiquark] of the modified operator $T_{2}$ mainly considers the effect of the quarkantiquark energy created in the vacuum, i.e., that it suppresses the contribution from meson-meson states with high energy. The damping factor $\exp \left(-R_{A V}{ }^{2} / R_{0}^{2}\right)$ takes into account the fact that the created quark-antiquark pair should not be far away from the source particle. The convergence problem was also studied in Ferretti and Santopinto's work, where only the contribution from the closest set of meson-meson intermediate states were calculated and the contributions from other states were taken as some kind of global constant [44]. With some reasonable arguments, the parameters $f$ and $R_{0}$ were fixed, and the parameter $\gamma$ was determined by fitting the decay width of $\rho \rightarrow \pi \pi$,

$$
\gamma=32.2 f=0.5 \mathrm{fm} \quad R_{0}=1.0 \mathrm{fm} .
$$

Based on the modified transition operator $T_{2}$, Chen et al. calculated the mass shift of a light meson and adjusted some parameters of the quark model to bring unquenched light ground-state meson masses into agreement with experimental data,

$$
\alpha_{0}=3.85, \quad \Delta=-58.5 .
$$

\section{UNQUENCHED QUARK MODEL}

The mass and structure of a meson in the unquenched quark model are obtained by solving the Schrödinger equation

$$
H \Psi_{I M_{I}}^{J M_{J}}=E \Psi_{I M_{I}}^{J M_{J}},
$$

where $\Psi_{I M_{I}}^{J M_{J}}$ is the unquenched wave function of the system which contains two- and four-quark components. It can be written as

$$
\Psi_{I M_{I}}^{J M_{J}}=c_{2} \Psi_{I M_{I}}^{J M_{J}}(2 q)+\sum_{i=1}^{N} c_{4 i} \Psi_{i, I M_{I}}^{J M_{J}}(4 q)
$$

where $\Psi(2 q)$ and $\Psi(4 q)$ are the wave functions with twoand four-quark components, respectively (the simplified symbols are used to save space), and $N$ is the total number of four-quark channels.

In the nonrelativistic quark model, the number of particles is conserved. So there is no rigorous way to write the Hamiltonian of the unquenched quark model. Here we only give a description of the Hamiltonian $H$ as follows:

$$
H=H_{2 q}+H_{4 q}+T_{24},
$$

where $H_{2 q}$ acts on the wave function of the quark-antiquark component $\Psi(2 q)$, and $H_{4 q}$ only acts on the wave function of the four-quark component $\Psi(4 q) . T_{24}$ takes the form of the transition operator in the ${ }^{3} P_{0}$ model [Eq. (15) or Eq. (20)], which mix the two- and four-quark components. So, in this way, the matrix elements of the Hamiltonian can be written as

$$
\begin{aligned}
\langle\Psi|H| \Psi\rangle= & c_{2}^{2}\left\langle\Psi(2 q)\left|H_{2 q}\right| \Psi(2 q)\right\rangle \\
& +\sum_{i, j=1}^{N} c_{4 i}^{*} c_{4 j}\left\langle\Psi_{i}(4 q)\left|H_{4 q}\right| \Psi_{j}(4 q)\right\rangle \\
& +\sum_{i=1}^{N} c_{4 i}^{*} c_{2}\left\langle\Psi_{i}(4 q)\left|T_{24}\right| \Psi(2 q)\right\rangle \\
& +\sum_{j=1}^{N} c_{2}^{*} c_{4 j}\left\langle\Psi(2 q)\left|T_{24}^{\dagger}\right| \Psi_{j}(4 q)\right\rangle .
\end{aligned}
$$

Then, we get block Hamiltonian and overlap matrices:

$$
\begin{array}{r}
(H)=\left[\begin{array}{ccccc}
\left\langle H_{2 q}\right\rangle & \left\langle H_{24}\right\rangle_{1} & \left\langle H_{24}\right\rangle_{2} & \ldots & \left\langle H_{24}\right\rangle_{n} \\
\left\langle H_{42}\right\rangle_{1} & \left\langle H_{4 q}\right\rangle_{11} & \left\langle H_{4 q}\right\rangle_{12} & \ldots & \left\langle H_{4 q}\right\rangle_{1 n} \\
\left\langle H_{42}\right\rangle_{2} & \left\langle H_{4 q}\right\rangle_{21} & \left\langle H_{4 q}\right\rangle_{22} & \ldots & \left\langle H_{4 q}\right\rangle_{2 n} \\
\ldots & \ldots & \ldots & \ldots & \ldots \\
\left\langle H_{42}\right\rangle_{n} & \left\langle H_{4 q}\right\rangle_{n 1} & \left\langle H_{4 q}\right\rangle_{n 2} & \ldots & \left\langle H_{4 q}\right\rangle_{n n}
\end{array}\right], \\
(N)=\left[\begin{array}{ccccc}
\left\langle N_{2 q}\right\rangle & 0 & 0 & \ldots & 0 \\
0 & \left\langle N_{4 q}\right\rangle_{11} & \left\langle N_{4 q}\right\rangle_{12} & \ldots & \left\langle N_{4 q}\right\rangle_{1 n} \\
0 & \left\langle N_{4 q}\right\rangle_{21} & \left\langle N_{4 q}\right\rangle_{22} & \ldots & \left\langle N_{4 q}\right\rangle_{2 n} \\
\ldots & \ldots & \ldots & \ldots & \ldots \\
0 & \left\langle N_{4 q}\right\rangle_{n 1} & \left\langle N_{4 q}\right\rangle_{n 2} & \ldots & \left\langle N_{4 q}\right\rangle_{n n}
\end{array}\right],
\end{array}
$$

where $\left\langle H_{2 q}\right\rangle,\left\langle H_{24}\right\rangle_{j}$, and $\left\langle H_{4 q}\right\rangle_{i j}$ are $\left\langle\Psi(2 q)\left|H_{2 q}\right| \Psi(2 q)\right\rangle$, $\left\langle\Psi(2 q)\left|T_{24}\right| \Psi_{j}(4 q)\right\rangle$, and $\left\langle\Psi_{i}(4 q)\left|H_{4 q}\right| \Psi_{j}(4 q)\right\rangle$, respectively. The subscript labels the index of the four-quark channel. By solving the generalized eigenequation

$$
\left((H)-E_{n}(N)\right)\left(C_{n}\right)=0,
$$

we get the eigenenergy $E_{n}$ and the expansion coefficients $C_{n}$.

\section{NUMERICAL RESULTS AND DISCUSSIONS}

In the present calculation, we focus on the charmonium state $\chi_{c 1}(2 P)$ and try to explain the well-known exotic state $X(3872)$ in the unquenched quark model. To fix the parameters associated with the charm quark, the two charmonia $\eta_{c}$ and $J / \psi$ are also investigated in the 
unquenched quark model. For comparison, two transition operators-the traditional one and the modified one-are used in the calculation.

\section{A. Accumulating approach}

Generally, the dimension of $H$ in the unquenched quark model is very big and the matrix construction process is very complex. So all the previous unquenched quark model calculation adopt an accumulating approach, that is to do two-channel coupling calculation, bare state $\Psi(2 q)$ and one of the four-quark state $\Psi_{i}(4 q)$, to get the mass shift $\Delta m_{i}$ and the probability fraction of four-quark component $P_{i}$, then the total mass shift $\Delta M_{t}$ and the total fraction $P_{t}$ of four-quark component are obtained by accumulating $\Delta m_{i}$ and $P_{i}$,

$$
\begin{aligned}
\Delta M_{t} & =\sum_{i=1}^{N} \Delta m_{i}, \\
P_{t} & =\sum_{i=1}^{N} P_{i} .
\end{aligned}
$$

In this approach, the dimension of the Hamiltonian matrix $H$ needs to be diagonalized becomes much smaller. The approach works well if the cross matrix elements between different four-quark channels are small enough. Unfortunately, this is not always the case. If there is a coupling between two four-quark channels, the accumulating approach will introduce errors, especially for the case when the energy of the four-quark state is close to the bare mass of the meson. We compare the results for the states $\eta_{c}$ and $\chi_{c 1}(2 P)$ between the accumulating approach and the full-channel diagonalization in Table II, where the

TABLE II. The mass shifts and probability fractions of fourquark components in the accumulating approach and the threechannel coupling calculation with the unmodified transition operator. $D \bar{D}^{*}\left(D_{s}^{*} \bar{D}_{s}\right)$ stands for the two-channel coupling $c \bar{c}-$ $D \bar{D}^{*}\left(c \bar{c}-D_{s}^{*} \bar{D}_{s}\right)$. "Total" is the sum of the two two-channel coupling results. $D \bar{D}^{*}+D_{s}^{*} \bar{D}_{s}$ denotes the three-channel coupling $c \bar{c}-D \bar{D}^{*}-D_{s}^{*} \bar{D}_{s}$. An " $S$ " in parentheses denotes that the relative motion between two clusters is in the $S$ wave.

\begin{tabular}{lcccc}
\hline \hline & \multicolumn{2}{c}{$\eta_{c}$} & \multicolumn{2}{c}{$\chi_{c 1}(2 P)$} \\
\hline Bare mass & 2986.28 & \multicolumn{2}{c}{3889.62} \\
Exp & 2980.30 & \multicolumn{2}{c}{3871.69} \\
Meson-meson & $\Delta m_{i}(\mathrm{MeV})$ & $P_{i}$ & $\Delta m_{i}(\mathrm{MeV})$ & $P_{i}$ \\
$D \bar{D}^{*}(S)$ & -189.75 & $4.02 \%$ & -73.65 & $43.09 \%$ \\
$D_{s}^{*} \bar{D}_{s}(S)$ & -76.50 & $1.75 \%$ & -15.13 & $1.26 \%$ \\
Total & -266.25 & $5.77 \%$ & -88.78 & $44.35 \%$ \\
$D \bar{D}^{*}+D_{s}^{*} \bar{D}_{s}(S)$ & -266.71 & $5.20 \%$ & -83.39 & $35.53 \%$ \\
$D \bar{D}^{*}(S)$ & -189.75 & $4.02 \%$ & -73.65 & $43.09 \%$ \\
$D^{*} \bar{D}(S)$ & -189.75 & $4.02 \%$ & -73.65 & $43.09 \%$ \\
Total & -379.50 & $8.04 \%$ & -147.30 & $86.18 \%$ \\
$D \bar{D}^{*}+D^{*} \bar{D}(S)$ & -369.43 & $6.4 \%$ & -117.11 & $33.38 \%$ \\
\hline \hline
\end{tabular}

TABLE III. The mass shift of $\eta_{c}(1 S)$ in the different calculations (in units of $\mathrm{MeV}$ ).

\begin{tabular}{lcccc}
\hline \hline Meson-meson & Ref. [16] & Ref. [17] & Ref. [45] & This work \\
\hline$D \bar{D}^{*}\left(D^{*} \bar{D}\right)$ & -59 & -34 & -114 & -189.75 \\
$D^{*} \bar{D}^{*}$ & -55 & -31 & -105 & -354.48 \\
$D_{s} \bar{D}_{s}^{*}\left(D_{s}^{*} \bar{D}_{s}\right)$ & -26 & -8 & -106 & -76.50 \\
$D_{s}^{*} \bar{D}_{s}^{*}$ & -35 & -8 & -98 & -147.14 \\
\hline \hline
\end{tabular}

traditional transition operator $T_{1}$ is used. There is no coupling between $D \bar{D}^{*}$ and $D_{s} \bar{D}_{s}^{*}$, so the mass shifts and the probability fractions of the four-quark components for $\eta_{c}$ and $\chi_{c 1}(2 P)$ from the accumulating approach are almost the same as those from three-channel diagonalization. However, if there is a coupling between $D \bar{D}^{*}$ and $D^{*} \bar{D}$, then the different approaches will give different results. For $\eta_{c}$, the accumulating approach overestimates the mass shift by about $10 \%$, and the probability fraction by about $30 \%$. For $\chi_{c 1}(2 P)$, the overestimates are $30 \%$ for the mass shift and $160 \%$ for the probability fraction, because $D \bar{D}^{*}$ is an open channel for the state $\chi_{c 1}(2 P)$. Thus, here the multichannel coupling calculation is adopted if there are nonzero matrix elements between different four-quark channels.

We compare our results for $\eta_{c}$ and those from other calculations in Table III, where the same transition operator $T_{1}$ is employed. Our calculation clearly gives larger mass shifts than those found in other works. The reason is that different wave functions are used in the different calculations. In most previous works $[16,17,45]$ the SHO wave functions were chosen as the radial part of the orbital wave functions of mesons, and the relative-motion wave function between two meson clusters was chosen as the plane wave function. The plane-wave approximation is a good one in the decay calculation, but it would not be a good one for the bound-state calculation. In most cases the SHO wave function can describe the ground meson well, but different parameter values should be used for different mesons. To simplify the calculations, the same parameter values were used for different mesons in previous works. In our calculation, all of the wave functions are determined by system dynamics. In this way, we are able to perform a selfconsistent calculation.

\section{B. Results}

The direct use of the transition operator $T_{1}$ leads to rather large mass shifts for ground-state mesons, and violates the validity of the quark model description of ground-state mesons, especially for light mesons [32]. The transition operator has to be modified in the channel coupling calculation. In the present work, the modified operator $T_{2}$ is employed. Using the modified operator, and keeping all of the parameters used in the valence quark model unchanged, we calculate the mass shifts and probability fractions of the four-quark components of the state $\chi_{c 1}(2 P)$. 
TABLE IV. The mass shifts and probability fractions of fourquark components of $\chi_{c 1}(2 P)$ (in units of $\mathrm{MeV}$ ). The " $S$ " and " $D$ " in parentheses denote that the relative motion between two clusters is in the $S$ wave and/or $D$ wave.

\begin{tabular}{lccccc}
\hline \hline \multirow{2}{*}{ Transition operator } & \multicolumn{2}{c}{$T_{1}$} & & \multicolumn{2}{c}{$T_{2}$} \\
\cline { 2 - 3 } \cline { 5 - 6 } States & $\Delta m_{i}$ & $P_{i}$ & & $\Delta m_{i}$ & $P_{i}$ \\
\hline$D \bar{D}^{*}+D^{*} \bar{D}(S+D)$ & -202.72 & $47.14 \%$ & -76.37 & $26.76 \%$ \\
$D_{s} \bar{D}_{s}^{*}+D_{s}^{*} \bar{D}_{s}(S+D)$ & -54.87 & $4.1 \%$ & -8.84 & $1.98 \%$ \\
$D^{*} \bar{D}^{*}(D)$ & -201.34 & $10.32 \%$ & -85.61 & $6.42 \%$ \\
$D_{s}^{*} \bar{D}_{s}^{*}(D)$ & -68.03 & $2.01 \%$ & -10.39 & $0.72 \%$ \\
Total & -526.96 & $63.57 \%$ & -181.21 & $36.88 \%$ \\
Bare mass & \multicolumn{4}{c}{3889.62} \\
Unquenched mass & 3362.66 & 3871.69 & \\
Exp & & 3708.41 & \\
\hline \hline
\end{tabular}

The results are listed in Table IV. For comparison, the results with the unmodified operator $T_{1}$ are also listed in Table IV. From the table, we can see that the mass shifts with $T_{2}$ are about $1 / 3$ of the mass shifts with $T_{1}$. For the results with $T_{2}$, the open channel $D \bar{D}^{*}$ (both the $S$ and $D$ waves of the relative motion between $D$ and $\bar{D}^{*}$ are considered) makes the largest contribution, and pushes the bare mass of $\chi_{c 1}(2 P)$ down about $76.37 \mathrm{MeV}$. The $D$ wave $D^{*} \bar{D}^{*}$ also pushes the bare mass down about $85.61 \mathrm{MeV}$. The mass shifts from the states $D_{s} \bar{D}_{s}^{*}$ and $D_{s}^{*} \bar{D}_{s}^{*}$ are small. Finally, coupling of all the four-quark components make mass shifts $-181.21 \mathrm{MeV}$ to the state $\chi_{c 1}(2 P)$, and the unquenched mass of the state is $3708.41 \mathrm{MeV}$. In the valence quark model, the parameters are determined by fitting the ground-state mesons. In UQM, the parameters have to be readjusted to fit the meson spectrum.
TABLE V. Adjusted quark model parameters.

\begin{tabular}{lcccc}
\hline \hline Parameter & & ChQM & Ref. [32] & This work \\
\hline Quark masses & $m_{c}(\mathrm{MeV})$ & 1728 & 1728 & 1690.5 \\
Confinement & $a_{c}\left(\mathrm{MeV} \mathrm{fm}^{-2}\right)$ & 101 & 101 & 112.7 \\
& $\Delta(\mathrm{MeV})$ & -78.3 & -58.3 & -52.7 \\
$\alpha_{s}$ & $\alpha_{q q}$ & 0.57 & 0.60 & 0.60 \\
& $\alpha_{q s}$ & 0.54 & 0.56 & 0.56 \\
& $\alpha_{q c}$ & 0.49 & 0.52 & 0.52 \\
& $\alpha_{s c}$ & 0.44 & 0.46 & 0.46 \\
& $\alpha_{c c}$ & 0.38 & 0.39 & 0.39 \\
\hline \hline
\end{tabular}

If we want to reproduce the mass of the state $X(3872)$ exactly, we can fine-tune the model parameters related to the charm quark and keep the light meson sector unchanged as much as possible. To justify the fine-tuning, the charmonia $\eta_{c}$ and $J / \psi$ are also calculated in the unquenched quark model. The adjusted parameters are listed in Table $\mathrm{V}$ with the original parameters and the parameters adjusted in Ref. [32]. From the table, we can see that the adjustment of parameters is small, less than 5\% except the energy shift $\Delta$.

The results with the new parameters for the charmonia $\eta_{c}$, $J / \psi$, and $\chi_{c 1}(2 P)$ are shown in Table VI. The masses of $\eta_{c}$ and $J / \psi$ are fitted by adjusting the parameters, so the experimental data are reproduced well. The dominant components of $\eta_{c}$ and $J / \psi$ are $c \bar{c}$, over $90 \%$. In this case the calculated unquenched mass of $\chi_{c 1}(2 P)$ is $3871.7 \mathrm{MeV}$, which is almost the experimental value for $X(3872)$. In our calculation, the state is a mixture $c \bar{c}$ and four-quark components. The dominant component is still $c \bar{c}(\sim 70 \%)$, the fraction of $D \bar{D}^{*}+D^{*} \bar{D}$ is around $22.5 \%, D^{*} \bar{D}^{*}, 5.6 \%$ and $D_{s}^{(*)} \bar{D}_{s}^{(*)}, 2.4 \%$. The results are qualitatively consistent with some previous works $[11,17,20]$. However, because of

TABLE VI. The mass shifts and probability fractions of four-quark components in UQM. ' $S$, ' $P$ ' and ' $D$ ' in the parentheses denotes the relative motion between two clusters are in $S$-, $P$ - and $D$-wave.

\begin{tabular}{|c|c|c|c|c|c|c|}
\hline \multirow{2}{*}{$\begin{array}{l}\text { State } \\
\text { Bare mass } \\
\text { Meson-meson state }\end{array}$} & \multicolumn{2}{|c|}{$\begin{array}{c}\eta_{c} \\
3047.0\end{array}$} & \multicolumn{2}{|c|}{$\begin{array}{c}J / \psi \\
3169.7\end{array}$} & \multicolumn{2}{|c|}{$\begin{array}{c}\chi_{c 1}(2 P) \\
4022.2\end{array}$} \\
\hline & $\Delta m_{i}$ & $P_{i}$ & $\Delta m_{i}$ & $P_{i}$ & $\Delta m_{i}$ & $P_{i}$ \\
\hline$D \bar{D}^{*}+D^{*} \bar{D}(P)$ & -26.3 & $2.5 \%$ & -20.5 & $2.2 \%$ & $\ldots$ & $\ldots$ \\
\hline$D_{s} \bar{D}_{s}^{*}+D_{s}^{*} \bar{D}_{s}(P)$ & -6.0 & $0.5 \%$ & -4.6 & $0.4 \%$ & $\ldots$ & $\ldots$ \\
\hline$D^{*} \bar{D}^{*}(P)$ & -24.8 & $2.2 \%$ & $\ldots$ & $\ldots$ & $\ldots$ & $\ldots$ \\
\hline$D_{s}^{*} \bar{D}_{s}^{*}(P)$ & -5.9 & $0.5 \%$ & $\ldots$ & $\ldots$ & $\ldots$ & $\ldots$ \\
\hline$D \bar{D}+D^{*} \bar{D}^{*}(P)$ & $\ldots$ & $\ldots$ & -39.4 & $4.0 \%$ & $\ldots$ & $\ldots$ \\
\hline$D_{s} \bar{D}_{s}+D_{s}^{*} \bar{D}_{s}^{*}(P)$ & $\ldots$ & $\ldots$ & -9.0 & $0.8 \%$ & $\ldots$ & $\ldots$ \\
\hline$D \bar{D}^{*}+D^{*} \bar{D}(S+D)$ & $\ldots$ & $\ldots$ & $\ldots$ & $\ldots$ & -68.2 & $22.5 \%$ \\
\hline$D_{s} \bar{D}_{s}^{*}+D_{s}^{*} \bar{D}_{s}(S+D)$ & $\ldots$ & $\ldots$ & $\ldots$ & $\ldots$ & -8.2 & $1.7 \%$ \\
\hline$D^{*} \bar{D}^{*}(D)$ & $\ldots$ & $\ldots$ & $\ldots$ & $\ldots$ & -63.9 & $5.6 \%$ \\
\hline$D_{s}^{*} \bar{D}_{s}^{*}(D)$ & & $\ldots$ & $\ldots$ & & -10.2 & $0.7 \%$ \\
\hline Total & -63.0 & $5.7 \%$ & -73.5 & $7.4 \%$ & -150.5 & $30.5 \%$ \\
\hline Unquenched mass & 2984.0 & $94.3 \%$ & 3096.2 & $92.6 \%$ & 3871.7 & $69.5 \%$ \\
\hline Exp & 2980.3 & & 3096.9 & & 3871.7 & \\
\hline
\end{tabular}


the modified transition operator, the contribution from the high Fock components are all suppressed, and the dominant component is still the valence one, which implied that the quenched quark model is a good zero-order approximation of the hadron spectrum. In other works, the dominant components of $\chi_{c 1}$ were meson-meson ones, the fraction of $c \bar{c}$ is small, $7 \%$ to $32 \%$ in Ref. [36], $7.5 \%$ to $11.2 \%$ in Ref. [46] and $14.7 \%$ in Ref. [44]. Kalashnikova got a little large fraction of $c \bar{c}, 54.3 \%$ [16].

\section{SUMMARY}

We developed an unquenched quark model to describe ordinary and exotic mesons in one framework. As this is a preliminary work, only four-quark components were taken into account, and the four-quark components were limited to meson-meson states. The transition operator is required to relate the valence part to the high Fock components. Here a modified version of the transition operator of the ${ }^{3} P_{0}$ model was employed. The modification consists of two parts. One is the fact that the creation probability will decrease when the energy of the created quark-pair increases. Another requires that the created quark cannot be far away from the valence quark pair. To minimize the error from the calculation, a powerful method for dealing with few-body systems - GEM - was used to find all of the necessary wave functions. The unquenched quark model was applied to the light meson spectrum, and a reasonable result was obtained. All of the mass shifts are around 15\% of the bare masses of the states. In this way, the valence quark model still successfully describes the low-lying meson spectrum. We also applied the model to the charmonium states in an attempt to explain the exotic state $X(3872)$.

By keeping the model parameters related to the light meson unchanged and fine-tuning the parameters related to the charm quark, we obtained an unquenched mass for $\chi_{c 1}(2 P)$ that is very close to the experimental value for $X(3872)$. At the same time, the masses of the charmonia $\eta_{c}$ and $J / \psi$ are reproduced well. In our UQM, the high Fock components of the ground-state charmonia $\eta_{c}$ and $J / \psi$ are small (less than 10\%). Similar results were obtained for the light mesons [32]. For the states which can decay strongly to two mesons, the probability fractions of the two-meson continua will be large, such as the $\pi \pi$ continua for the $\rho$ meson. Here we obtained similar results for $X(3872)$. The fraction of the two-meson continua is around $30 \%$. However, the dominant component of $X(3872)$ is still $c \bar{c}(70 \%)$.

To develop the unquenched quark model, the problem of convergence must be taken into account. Using the original transition operator of the ${ }^{3} P_{0}$ model and summing over infinite towers of the intermediate states, the results may not converge due to that there is no suppression of the contribution from the high lying states. Here we modified the transition operator to suppress the contribution from the high lying states, and convergent results were obtained. This also validates the valence quark model description of ground-state hadrons. All of the wave functions used in the calculation were obtained by solving the corresponding Schrödinger equation, and the mass shifts were obtained by solving a few-body problem. In this sense, our unquenched quark model calculation is a realistic and self-consistent one.

From our calculation we can see that the unquenched quark model is a promising phenomenological method to unify the descriptions of ordinary mesons and exotic mesons. Of course, further improvements are still needed, as the four-quark components may be hidden-color states or diquark-antidiquark states. To give a realistic description of all mesons the model parameters will need to be adjusted. We will address these problems in our future work.

\section{ACKNOWLEDGMENTS}

This work is supported partly by the National Natural Science Foundation of China under Contract Nos. 11775118, 11847145 and 11535005.
[1] S. K. Choi et al. (Belle Collaboration), Phys. Rev. Lett. 91, 262001 (2003).

[2] D. Acosta et al. (CDFII Collaboration), Phys. Rev. Lett. 93, 072001 (2004).

[3] V. M. Abazov et al. (D0 Collaboration), Phys. Rev. Lett. 93, 162002 (2004).

[4] B. Aubert et al. (BABAR Collaboration), Phys. Rev. D 71, 071103 (2005).

[5] T. Barnes and S. Godfrey, Phys. Rev. D 69, 054008 (2004).

[6] E. S. Swanson et al. Phys. Lett. B 598, 197 (2004).

[7] N. A. Tornqvist, Phys. Lett. B 590, 209 (2004).
[8] M. Suzuki, Phys. Rev. D 72, 114013 (2005).

[9] S. Pakasa and M. Suzuki, Phys. Lett. B 579, 67 (2004).

[10] B. Aubert et al. (BABAR Collaboration), Phys. Rev. Lett. 102, 132001 (2009).

[11] N. N. Achasov, Phys. Part. Nucl. 48, 839 (2017); EPJ Web Conf. 212, 02001 (2019).

[12] K. Abe et al. (Belle Collaboration), arXiv:hep-ex/0505038.

[13] E. S. Swanson, Phys. Lett. B 588, 189 (2004).

[14] Y. R. Liu, X. Liu, W. Z. Deng, and S. L. Zhu, Eur. Phys. J. C 56, 63 (2008). 
[15] C. E. Thomas and F. E. Close, Phys. Rev. D 78, 034007 (2008).

[16] Y. S. Kalashnikova, Phys. Rev. D 72, 034010 (2005).

[17] J. Ferretti and G. Galatà, and E. Santopinto, Phys. Rev. C 88, 015207 (2013).

[18] P. G. Ortega, J. Segovia, D. R. Entem, and F. Fernández, Phys. Rev. D 81, 054023 (2010).

[19] M. Cardoso, G. Rupp, and E. V. Bevern, Eur. Phys. J. C 75, 26 (2015).

[20] X. W. Kang and J. A. Oller, Eur. Phys. J. C 77, 399 (2017).

[21] L. Micu, Nucl. Phys. B10, 521 (1969).

[22] A. L. Yaouanc, L. Oliver, O. Pène, and J. C. Raynal, Phys. Rev. D 8, 2223 (1973).

[23] A. L. Yaouanc, L. Oliver, O. Pène, and J.-C. Raynal Phys. Rev. D 9, 1415 (1974).

[24] R. Bijker and E. Santopinto, Phys. Rev. C 80, 065210 (2009).

[25] E. Santopinto and R. Bijker, Phys. Rev. C 82, 062202(R) (2010).

[26] J. Ferretti, G. Galatá, E. Santopinto, and A. Vassallo, Phys. Rev. C 86, 015204 (2012).

[27] J. Ferretti, E. Santopinto, and H. García-Tecocoatzi, J. Phys. Soc. Jpn. Conf. Proc. 10, 022007 (2016).

[28] E. Santopinto and J. Ferretti, EPJ Web Conf. 96, 01026 (2015).

[29] P. G. Ortega, J. Segovia, D. R. Entem, and F. Fernández, J. Phys. G 40, 065107 (2013).

[30] P. G. Ortega, J. Segovia, D. R. Entem, and F. Fernández, Phys. Rev. D 94, 114018 (2016).
[31] P. G. Ortega, D. R. Entem, and F. Fernández, Phys. Lett. B 778, 1 (2018).

[32] X. Chen, J. Ping, C. D. Roberts, and J. Segovia, Phys. Rev. D 97, 094016 (2018).

[33] J. Vijande, F. Fernández, and A. Valcance, J. Phys. G 31, 481 (2005).

[34] E. Hiyama, Y. Kino, and M. Kamimura, Prog. Part. Nucl. Phys. 51, 223 (2003).

[35] B. Q. Li, C. Meng, and K. T. Chao, Phys. Rev. D 80, 014012 (2009).

[36] P. G. Ortega, J. Segovia, D. R. Entem, and F. Fernández, Phys. Rev. D 81, 054023 (2010).

[37] E. S. Ackleh, T. Barnes, and E. S. Swanson, Phys. Rev. D 54, 6811 (1996).

[38] W. Roberts and B. Silvestre-Brac, Few-Body Syst. 11, 171 (1992).

[39] S. Capstick and N. Isgur, Phys. Rev. D 34, 2809 (1986).

[40] S. Capstick and W. Roberts, Phys. Rev. D 49, 4570 (1994).

[41] P. R. Page, Nucl. Phys. B446, 189 (1995).

[42] M. Jacob and G. C. Wick, Ann. Phys. (N.Y.) 7, 404 (1959); 281, 774 (2000).

[43] A. Le Yaouanc, L. Oliver, O. Pène, and J. C. Raynal Phys. Lett. B 72, 57 (1977).

[44] J. Ferretti and E. Santopinto, Phys. Lett. B 789, 550 (2019).

[45] T. Barnes and E. S. Swanson, Phys. Rev. C 77, 055206 (2008).

[46] S. Coito, G. Rupp, and E. van Beveren, Eur. Phys. J. C 73, 2351 (2013). 